\title{
Detección de anticuerpos séricos en toros inmunizados contra campylobacteriosis
}

\author{
Campero, C.M.'; Morrell, E. ${ }^{1}$; Morsella, C. ${ }^{1}$; Paolicchi, F. ; Cano, D. ${ }^{1}$; Lázaro, L. ${ }^{2}$ \\ ${ }^{1}$ Grupo de Sanidad Animal, Instituto Nacional de Tecnología Agropecuaria, INTA, \\ Casilla de Correo 276, Balcarce (7620), Argentina. ${ }^{2}$ Veterinario actividad privada. \\ E-mail: ccampero@balcarce.inta.gov.ar
}

\begin{abstract}
Resumen
Campero, C.M.; Morrell, E.; Morsella, C.; Paolicchi, F.; Cano, D.; Lázaro, L.: Detección de anticuerpos séricos en toros inmunizados contra campylobacteriosis. Rev. vet. 21: 1, 8-12, 2010. En el presente ensayo se evaluó la repuesta sérica vacunando 169 toros jóvenes para carne, vírgenes de 12-24 meses de edad, inmunizados con vacunas a célula entera de Campylobacter fetus. El trabajo se realizó en cuatro establecimientos libres de campylobacteriosis de la provincia de Buenos Aires (Argentina). Se utilizaron tres vacunas comerciales nacionales, conteniendo cepas inactivadas de C. fetus y sus subspecies: vacunas A $(\mathrm{n}=84), \mathrm{B}$ $(\mathrm{n}=19)$ y $\mathrm{C}(\mathrm{n}=58)$ (suspensión de C. fetus subsp. fetus, intermedius y venerealis, inactivadas con formol). Las vacunas A y B poseían como vehículo hidróxido de aluminio, la vacuna $\mathrm{C}$ presentaba adyuvante oleoso. También se utilizó una vacuna experimental (D) $(n=8)$ dual, oleosa, elaborada por el INTA Balcarce, conteniendo antígenos inactivados de C. fetus subsp. fetus, C. fetus subsp. venerealis y Tritrichomonas foetus. Todas las vacunas se aplicaron por vía subcutánea, dos dosis con intervalo de 21 días. Los animales se sangraron a los días 0 , 21, 42, 93 y 123 post primera dosis vacunal (DPV). Los sueros fueron procesados por ELISA indirecto. Los valores de absorbancia expresados en densidad óptica fueron transformados a valores ELISA (VE). La vacuna experimental dual D demostró un incremento importante en los VE de los anticuerpos séricos con respecto las vacunas $\mathrm{A}$, B y C $(\mathrm{p}<0,05)$. Las vacunas $\mathrm{B}$ y $\mathrm{C}$ tuvieron mejor respuesta a los 21 y 42 días DPV respecto a la vacuna $\mathrm{A}(\mathrm{p}<0,05)$. A los $93 \mathrm{DPV}$, los animales del grupo $\mathrm{C}$ tuvieron un débil incremento de $\mathrm{VE}$ en comparación con aquellos animales de los grupos A y B $(\mathrm{p}<0,05)$. El desempeño general tendió a ser mayor en los animales inmunizados con la vacuna experimental que aquellos del grupo de vacunas comerciales. Se observaron VE muy bajos en general en las vacunas comerciales utilizadas. El método ELISA fue adecuado para la evaluación de la respuesta inmune sistémica en los toros vacunados.
\end{abstract}

Palabras clave: bovino, campylobacteriosis, inmunización, vacunas.

\begin{abstract}
Campero, C.M.; Morrell, E.; Morsella, C.; Paolicchi, F.; Cano, D.; Lázaro, L.: Sera antibodies detection in bulls immunized against campylobacteriosis. Rev. vet. 21: 1, 8-12, 2010. Serological response induced by whole-cell vaccines against Campylobacter fetus administered to 169 beef virgin bulls, aged from 12 to 24 months, was evaluated. The assay was performed in four Campylobacter-free beef cattle farms from Buenos Aires, Argentina. Three commercial national inactivated vaccines against $C$. fetus were used. Vaccines A ( $\mathrm{n}=$ 84), B ( $\mathrm{n}=19)$ and $\mathrm{C}(\mathrm{n}=58)$ contained C. fetus subsp. fetus, intermedius and venerealis antigens. Adjuvant in vaccines $\mathrm{A}$ and $\mathrm{B}$ was aluminum hydroxide, whereas vaccine $\mathrm{C}$ contained an oil-based adjuvant. An experimental vaccine (D) $(\mathrm{n}=8)$ containing $C$. fetus subsp. fetus, $C$. fetus subsp. venerealis and Tritrichomonas foetus inactivated antigens in an oil-based adjuvant developed at INTA Balcarce was also used. Bulls were vaccinated twice subcutaneously at three-week intervals. Blood samples were collected at 0,21, 42, 93, and 123 days after first vaccine dose (FVD). Indirect ELISA was used to detect systemic $C$. fetus antibodies. Optical densities of the samples were converted to ELISA values (EV). Bulls of group D showed high $\mathrm{EV}$ values, with the highest systemic immune response compared to animals of groups A, B and $C(p<0.05)$, during all the sampling period. EV from animals injected with vaccines $A$, $\mathrm{B}$, and $\mathrm{C}$ demonstrated a better performance compared to vaccine $\mathrm{A}(\mathrm{p}<0.05)$. At 93 days $\mathrm{FVD}$, animals of group $\mathrm{C}$ had a lower increment of $\mathrm{EV}$ in comparison to those of groups $\mathrm{A}$
\end{abstract}


and $B(p<0.05)$. Immunization performance tended to be better in experimental rather than commercial vaccines, the later producing very low EV values. The ELISA method was adequate for the evaluation of the systemic immune response in vaccinated bulls.

Key words: cattle, campylobacteriosis, immunization, vaccine.

\section{INTRODUCCIÓN}

Las pérdidas gestacionales en bovinos son causadas por una variedad de agentes infecciosos que afectan a la economía de productores de todo el mundo $4,5,7,15$. La campylobacteriosis genital bovina permanece como problema en rodeos para carne y leche de numerosos países $6,16,18$. La enfermedad causa muerte del embrión, abortos y reducción de la fertilidad en bovinos ${ }^{7}$ ${ }^{16}$. Campylobacter fetus tiene dos subespecies conocidas, C. fetus subsp. venerealis (con tropismo genital exclusivo, solo se transmite por vía venérea) y $C$. fetus subsp. fetus (reside en el tracto intestinal de bovinos/ovinos y puede ocasionalmente encontrarse en la cavidad prepucial del toro por contaminación fecal) ${ }^{16}$. Existe además una variedad tolerante a la glicina denominada C. fetus subsp. venerealis biovar. intermedius; dicha tolerancia es debida a mutación o transducción de material genético ${ }^{19,24}$.

La diferenciación de las subespecies se basa en la caracterización fenotípica y genotípica. Las vaquillonas vírgenes son muy susceptibles a la infección, desarrollando endometritis, mortalidad embrionaria temprana y abortos en la mitad de la gestación 7, 9,23. Trabajos realizados por nuestro grupo permitieron identificar el rol que le cabe a C. fetus subsp. fetus como el agente más frecuentemente aislado de fetos abortados por campylobacteriosis en bovinos de la región ${ }^{7}$.

Es importante el desarrollo de vacunas para el control de las enfermedades de transmisión sexual en bovinos. La campylobacteriosis en los toros es crónica, subclínica, cursa sin afectar su libido ni fertilidad y es más frecuente en toros adultos y viejos ${ }^{5,9}$. La vacunación sistémica en toros arrojó resultados promisorios ${ }^{3,}$ ${ }^{20}$. Diferentes trabajos realizados sobre vacunación en hembras mostraron resultados satisfactorios ${ }^{14}$. Otros autores evidenciaron el rol de los anticuerpos protectores contra este organismo ${ }^{2,20}$. Las vacunas deben contener cepas autóctonas antigénicamente aptas; su importancia radica en que eliminan el estado de portador de la hembra ${ }^{1}$. Si bien existen vacunas nacionales e importadas para la profilaxis de la campylobacteriosis tanto para hembras como machos, la información sobre la eficiencia de estas vacunas a nivel nacional es escasa ${ }^{8}$ no existiendo información actualizada sobre la respuesta humoral a la vacunación en toros.

El objetivo de este trabajo fue evaluar en toros el comportamiento humoral de algunas vacunas comerciales y una vacuna experimental contra la campylobacteriosis.

\section{MATERIAL Y MÉTODOS}

Animales, vacunaciones y muestreos. El ensayo se realizó en cuatro establecimientos libres de campylobacteriosis ubicados en el sudeste de la Provincia de Buenos Aires (Argentina) durante los años 2003-2005. Se utilizaron 169 toros vírgenes, de 12 a 24 meses de edad, de razas Aberdeen Angus y Hereford, clínicamente aptos. Los animales mantuvieron muy buena condición corporal (6-7) durante todo el ensayo. Se utilizaron tres vacunas comerciales nacionales inactivadas, conteniendo cepas de C. fetus y sus subspecies. A continuación se detalla la composición de las vacunas según lo descripto en los marbetes comerciales al momento de su empleo: vacuna A (Campy 3, Laboratorio Rosenbusch, Serie: 07/02, dosis: $2 \mathrm{ml}$ subcutánea, composición: C. fetus subsp. fetus, intermedius y venerealis, inactivadas con formol en vehículo de hidróxido de aluminio), vacuna B (Vibriosan, Laboratorio Sanidad Ganadera, Serie 2306, dosis: $3 \mathrm{ml}$ subcutánea, suspensión de C. fetus subs. fetus, intermedius y venerealis, inactivadas con formol en gel de hidróxido de aluminio como adyuvante), vacuna C (Campylobacter 40, Laboratorio Centro Diagnóstico Veterinario SA, Serie 003, dosis: $5 \mathrm{ml}$ subcutánea, composición: $40 \mathrm{mg}$ por dosis de C. fetus subsp. fetus, intermedius y venerealis, inactivados y con adyuvante oleoso Marcol 52, Arlacel C y Tween 80). Finalmente, se usó la vacuna D dual experimental, oleosa (Marcol 52, Span 80) (40\% aceite, $60 \%$ antígeno en fase acuosa), inactivado, elaborada en el INTA Balcarce con cepas de C. fetus subsp. fetus, $C$. fetus subs. venerealis (30 mg peso seco de antígeno de C. fetus $\mathrm{x}$ dosis vacunal) y $T$. foetus (Campero et al., sin publicar). En todos los casos, las vacunas se aplicaron mediante dos dosis por vía subcutánea en el lado izquierdo de la tabla del cuello, con intervalo de 21 días y en volúmenes según indicaciones de los elaboradores (Tabla 1). Las vacunas comerciales fueron adquiridas directamente de veterinarias de la zona. Se realizaron muestreos sanguíneos, a saber: a los días $0,21,42,93$ 
y 123 post primera dosis vacunal (DPV). Los sueros se mantuvieron a $-20^{\circ} \mathrm{C}$ hasta el momento de su uso.

Test de ELISA. Los sueros fueron analizados mediante el test de ELISA indirecto según lo descripto por Hum et al. ${ }^{18}$ con mínimas modificaciones ${ }^{12}$. El antígeno utilizado fue obtenido mediante sonicación de cultivos líquidos de C. fetus subsp. venerealis, C. fetus subsp. venerealis biotipo intermedius y $C$. fetus subsp. fetus, cepas tipificadas por pruebas bioquímicas, PCR y AFLP. Las placas de poliestireno (Immulon 1B x 96 pocillos) fueron sensibilizadas con los antígenos específicos previamente mencionados y adicionados los sueros problemas y los sueros controles positivos y negativos respectivos, previamente diluidos (dilución óptima previa titulada 1:500) en solución salina bufferada (SSB) con $0.05 \%$ de Tween 20 y gelatina al $0,2 \%$ (SSB-TwG). Las placas se lavaron con SSB-TwG (x3). Luego se utilizó antisuero IgG bovina conjugado con peroxidasa (Whole IgG rabbit anti-bovine, Sigma, St. Louis, Mo, USA) a la dilución óptima de 1:2000 en SSB-TwG. Se empleó como cromógeno el ácido 5-aminosalicílico haciendo la lectura de las placas en un espectrofotómetro (Multiskan Thermo Labsystem, Finlandia) a través de un filtro de $450 \mathrm{~nm}$.

Análisis estadístico. Se utilizó el límite confianza del 95\% $(\mathrm{p}<0,05)$. Los valores de absorbancia expresados en densidad óptica fueron transformados a valores ELISA (VE) cuyos promedios se graficaron. Los datos promedios de los VE fueron analizados mediante test de varianza para medidas repetidas en el tiempo utilizando el procedimiento MIXED del SAS ${ }^{13,21}$.

\section{RESULTADOS}

Los resultados de la serología se expresaron como VE (Figura 1). Se observó que las vacunas B y C tuvieron mejor respuesta a los 21 y 42 días post primera dosis vacunal (DPV) respecto a la vacuna A. Por su parte, la vacuna experimental dual D demostró un incremento en los anticuerpos séricos, persistiendo los mismos elevados en los sucesivos muestreos, respecto al resto de las vacunas $(\mathrm{p}<0,05)$. Con esta vacuna experimental se observó una diferencia muy elevada con respecto a los VE obtenidos ya a los $42 \mathrm{DPV}$, llegando a valores próximos al máximo luego de la aplicación de la segunda dosis y persistiendo hasta el final del ensayo Las vacunas B y C tuvieron mejor respuesta a los 21 y 70 DPV con respecto a la vacuna $A(p<0,05)$. Se observó una mayor persistencia de títulos vacunales a los 110 DPV en los animales inmunizados con la vacuna $\mathrm{C}$ con respecto a los vacunados con A y B $(\mathrm{p}<0,05)$.

\section{DISCUSIÓN}

Este estudio señala la producción de anticuerpos séricos medidos por el método de Elisa indirecto en toros vacunados por vía sistémica con vacunas comerciales y una vacuna experimental, inactivadas y específicas, contra campylobacteriosis. Nuestro grupo ha participado en forma activa en los últimos años me-
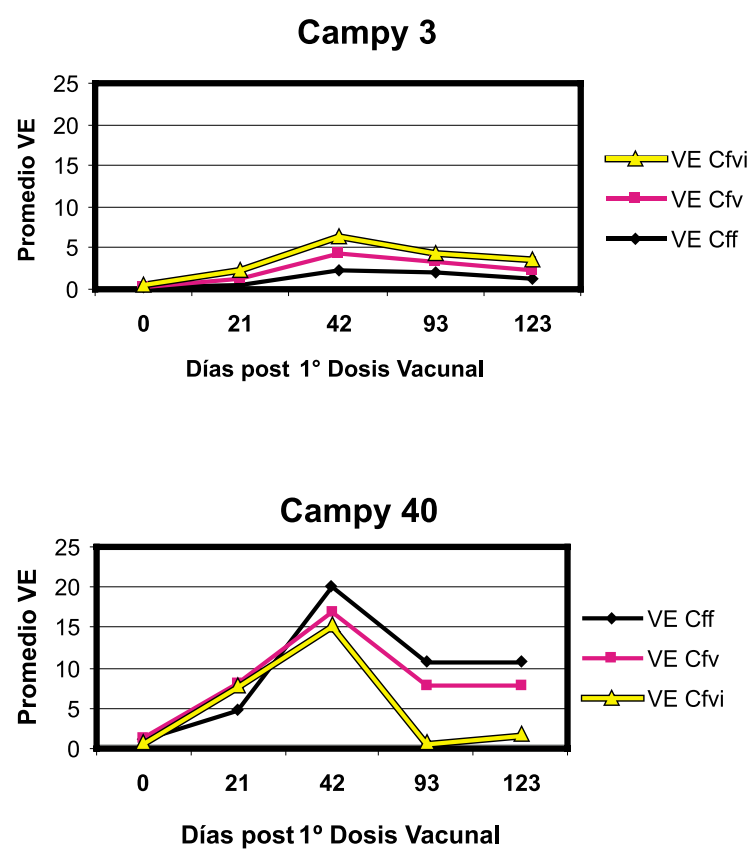
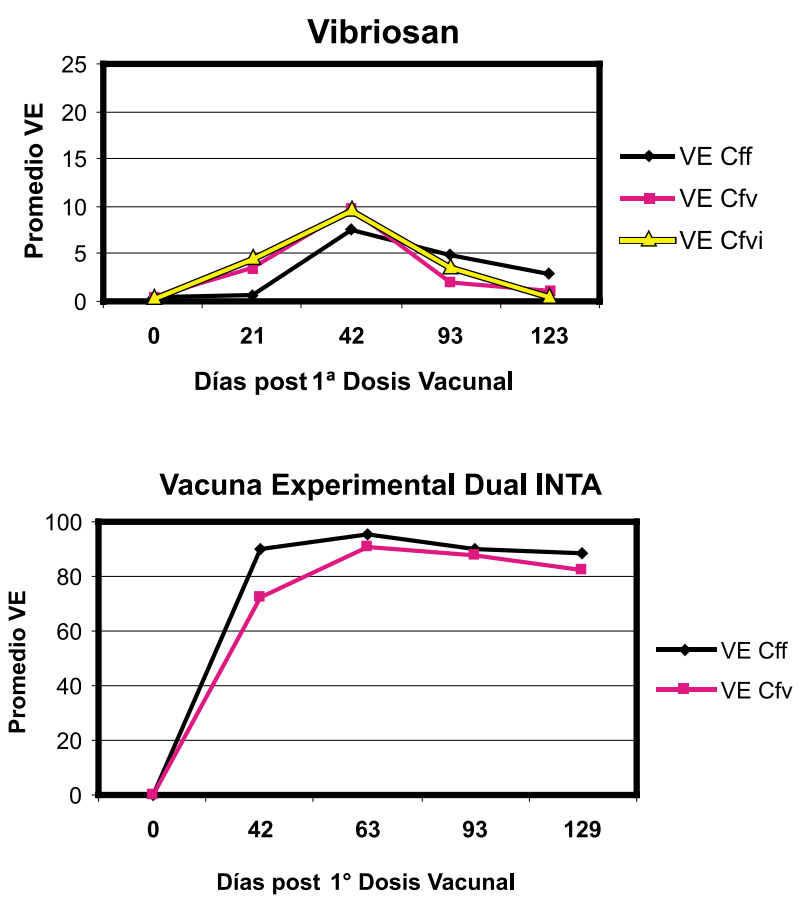

Figura 1. Anticuerpos séricos (valores Elisa promedio) a diferentes suspensiones de C. fetus según las vacunas utilizadas. 
diante ensayos en el medio para demostrar la eficacia de algunas vacunas comerciales contra campylobacteriosis en vaquillonas. En un trabajo previo efectuado en vaquillonas, se demostró que las vacunas comerciales se comportaron de manera similar y originaron un menor nivel de anticuerpos comparados con una vacuna experimental ${ }^{8}$.

Ensayos realizados por nuestro grupo con dos vacunas polivalentes comerciales (C. fetus fetus y venerealis, Leptospira pomona, virus IBR-BVD, $H$ somnus) en vaquillonas naturalmente desafiadas por servicio con toro infectado, confirmaron que ni las vaquillonas vacunadas ni los animales controles tuvieron un importante incremento en los niveles de anticuerpos sistémi$\cos ^{12}$. Si bien el número de animales utilizados fue bajo y el período de servicio corto, estos resultados sugieren una pobre inmunidad generada con este tipo de vacunas. Otros trabajos efectuados con vacunas duales experimentales elaboradas por nuestro grupo permitieron confirmar una adecuada respuesta sérica a $C$. fetus medida por Elisa (Campero et al. sin publicar) ${ }^{13}$. Los ensayos de vacunación en toros en nuestro medio los hicimos con una vacuna experimental en la década de $1980 \sin$ existir trabajos actualizados ${ }^{20}$.

El toro transmite y difunde la campylobacteriosis por el servicio natural o a través de la inseminación artificial con semen infectado ${ }^{15}$. La curación espontánea de toros jóvenes infectados suele ocurrir aunque el fenómeno es más raro en toros adultos 9,16 pudiendo atribuirse al escaso desarrollo de las criptas prepuciales ${ }^{5}$. Dado que C. fetus no es invasor tisular a nivel prepucial ni peniano, la respuesta inmune local es escasa y por ende, la infección suele ser crónica, especialmente en toros de $\geq 4$ años de edad ${ }^{5}$. La inmunización sistémica contra C. fetus estimula la producción de anticuerpos séricos y locales hacia antígenos de superficie del organismo $^{1,22}$. Las vacunas oleosas estimulan una sólida respuesta sistémica a la IgG1 e IgG2 y también similares anticuerpos en la vagina y útero ${ }^{14}$. Es por ello que se asume que la inmunización parenteral es importante para evitar la infección ${ }^{10,11}$, pérdidas reproductivas ${ }^{8} \mathrm{y}$ aún podrá curar la infección en hembras ${ }^{22}$.

En el presente trabajo, el empleo de 3 vacunas comerciales (dos con vehículo en hidróxido de aluminio y la tercera en vehículo oleoso) aplicadas en forma sistémica no incrementaron en forma marcada los anticuerpos sistémicos. Considerando el bajo número de animales utilizados y la falta de los desafíos intraprepuciales con cepas activas de C. fetus, hace que solamente podamos juzgar en forma parcial los resultados obtenidos. Por ende, no se pretende con este trabajo ni validar ni invalidar ninguno de los productos comerciales/experimental empleados. Si bien la inmunidad local a nivel prepucial no se determinó en este trabajo, la inmunidad sistémica estimulada por las vacunas comerciales analizadas no tuvo una adecuada respuesta sérica.

La menor antigenicidad de las vacunas comerciales frente a la vacuna experimental dual elaborada por el INTA Balcarce, se debería probablemente a la calidad y cantidad de antígeno en su composición. El efecto del adyuvante oleoso resulta de interés dado que las dos vacunas oleosas (C comercial y D experimental) tuvieron los mayores VE promedios con respecto a las otras dos vacunas comerciales. Sin embargo, se desconoce si dichos títulos son suficientemente protectores para prevenir la enfermedad. El inconveniente de los adyuvantes oleosos (usualmente se suele utilizar la combinación de Marcol 52, Arlacel C y Tween 80) en vacunas para bovinos suele ser la presencia de diferentes grados de granulomas locales en el área de aplicación $(3 \times 4 \mathrm{~cm})$ y la mayor probabilidad que se presenten fenómenos de anafilaxia post vacunación.

La vacuna $B$ actualmente se elabora con adyuvante oleoso (Vibriosan oleoso) con similar composición de subespecies de C. fetus mencionadas previamente, por lo que sería de esperar una mejor respuesta antigénica motivada por el cambio de adyuvante, mientras que la vacuna $\mathrm{C}$ actualmente sigue teniendo vehículo oleoso pero posee solo dos subespecies de C. fetus en su composición. Trabajos previos utilizando el hidróxido de aluminio evidenciaron que el mismo induce una inmunidad menos duradera y debería ser administrado como máximo a los $10-15$ días previos al servicio ${ }^{1}$.

Las vacunas A y B no mencionan la cantidad de antígeno (mg de masa antigénica) en sus formulaciones comerciales. Trabajos previos con vacunas comerciales mono o trivalentes a $C$. fetus tuvieron pobre desempeño en vaquillonas porque no fueron elaboradas con cepas regionales ${ }^{8}$. Existen referencias sobre los escasos resultados al vacunar toros crónicamente infectados pese a que fueron vacunados en dos ocasiones y tuvieron altos títulos séricos por ELISA ${ }^{17}$. La presencia de un peso seco antigénico mínimo es de importancia al evaluar una vacuna ${ }^{11}$. Trabajos experimentales en vacunas contra campylobacteriosis comprobaron que $40 \mathrm{mg}$ de antígeno por dosis en vehículo oleoso generaron buena protección contra infecciones genitales en hembras ${ }^{8,11}$. La selección de cepas aptas de C. fetus para la elaboración de vacunas es primordial para una buena inmunidad.

El test de ELISA indirecto usado es una buena herramienta para medir la respuesta inmune sistémica en bovinos vacunados con C. fetus. La insuficiente información específica provista por algunos elaboradores y la ausencia de ensayos en bovinos crea un cierto grado de escepticismo sobre la eficacia de dichas vacunas en bovinos desafiados o su empleo en rodeos infectados con campylobacteriosis. Se necesitan otros trabajos ampliatorios que aporten información adicional para establecer la efectividad de las vacunas comerciales en condiciones de infección natural.

Agradecimientos. Nuestro sincero agradecimiento a los propietarios y al personal de campo de los establecimientos Flores Chicas y La Cerradilla por su importante apoyo para realizar parte de este ensayo. A personal auxiliar de las reservas ganaderas 6 y 7 del INTA Balcarce por su apoyo en las tareas de muestreo de los animales. 


\section{REFERENCIAS}

1. Berg RL, Firehammer BD. 1978. Effect of interval between booster vaccination and time of breeding on protection against campylobacteriosis (vibriosis) in cattle. $J A V$ MA 173: 467-471.

2. Berg R, Firehammer B, Border M, Myers L. 1979. Effects of passively and actively acquired antibody on bovine Campylobacteriosis (vibriosis). Am J Vet Res 40: 21-25.

3. Bouters R, De Keyser J, Vandeplassche M, Van Aert A, Brone E, Bonte P. 1973. Vibrio fetus infection in bulls: curative and preventive vaccination. Br Vet J 129: 52-57.

4. Campero CM. 2000. Las enfermedades reproductivas de los bovinos: ayer y hoy. Anales Acad Nac Agron Vet 53: 88-112.

5. Campero CM. 2005. Consideraciones sobre la tricomoniasis y campylobacteriosis bovina. Rev Col Vet Prov Bs As 32: 47-51.

6. Campero CM, Ballabene N, Cipolla A, Zamora A. 1987. Dual infection in bulls with campylobacteriosis and trichomoniasis: treatment with dimetridazole chlorhydrate. Aust Vet J 64: 320-321.

7. Campero CM, Moore DP, Odeón AC, Cipolla AL, Odriozola E. 2003. Aetiology of bovine abortion in Argentina. Vet Res Comm 27: 359-369.

8. Cipolla AL, Campero CM, Morsella CG, Cano D, Caracino MJ, Marcone J. 1997. Vacunación contra campylobacteriosis en vaquillonas. Anales $25^{\circ}$ Jorn Urug Buiatr (Paysandú, Uruguay) p. 1-3.

9. Clark BL. 1971. Review of bovine vibriosis. Aust Vet J 47: 103-107.

10. Clark B, Dufty J, Monsbourgh M. 1968. Experimental Vibrio fetus (venerealis) infection in heifers. The immunizing properties of killed organisms injected subcutaneously. Aust Vet J 44: 110-114.

11. Clark BL, Dufty JH, Monsbourgh MJ, Parsonson IM. 1977. A dual vaccine for the immunization of cattle against vibriosis. Aust Vet J 53: 465-466.

12. Cobo E, Cipolla A, Morsella C, Cano D, Campero C. 2003. Effect of two commercial vaccines to Campylobacter fetus subspecies on heifers naturally challenged. $J$ Vet Med B 50: 75-80.
13. Cobo ER, Morsella C, Cano D, Cipolla A, Campero CM. 2004. Immunization in heifers with dual vaccines containing Tritrichomonas foetus and Campylobacter fetus antigens using systemic and mucosal routes. Theriogenology 62: $1367-1382$.

14. Corbeil LB, Schurig GD, Duncan JR, Wilkie BN, Winter AJ. 1981. Immunity in the female bovine reproductive tract based on the response to Campylobacter fetus. $A d v$ Exp Med Biol 137: 729-743.

15. Eaglesome M, Garcia M. 1992. Microbial agents associated with bovine genital tract infections and semen. Part I. Brucella abortus, Leptospira, Campylobacter fetus and Tritrichomonas foetus. Vet Bull 62: 743-775.

16. Garcia MM, Brooks BW. 1993. Campylobacter. In: Pathogenesis of bacterial infections in animals (Gyles CL, Thoen CO Ed), 2nd ed, Iowa State University, Ames, p. 262-272.

17. Hum S, Brunner J, Gardiner B. 1993. Failure of therapeutic vaccination of a bull infected with Campylobacter fetus. Aust Vet J 70: 386-387.

18. Hum S, Quinn C, Kennedy D. 1994. Diagnosis of bovine venereal campylobacteriosis by ELISA. Aust Vet J 71: 140-143.

19. On SL. 1996. Identification methods for campylobacters, helicobacters, and related organisms. Clin Microbiol Rev 9: 405-422.

20. Palladino MR, Campero CM. 1983. Vacunación contra vibriosis bovina en toros. Aspectos terapéuticos e inmunitarios. Gac Vet 45: 1036-1051.

21. SAS Institute Inc. 1987. SAS/STAT. Guide for personal computers. Version 6 Ed, Cary, NC, p. 320-392.

22. Schurig G, Hall G, Corbeil L, Duncan J, Winter A. 1975. Bovine venereal vibriosis: cure of genital infection in females by systemic immunization. Infect Immun 11: 245-251.

23. Skirrow MB. 1994: Diseases due to Campylobacter, Helicobacter and related bacteria. J Comp Pathol 111: 113149.

24. Smibert RM. 1978. The genus Campylobacter. Ann Rev Microbiol 32: 673-709. 